\title{
Effects of Air and Water Contents on the Effective Thermal Conductivity of Air-impregnated Gels
}

\author{
Takaharu SAKIYAma and Toshimasa YANO \\ Department of Agricultural Chemistry, Faculty of Agriculture, \\ The University of Tokyo, 1-1-I Yayoi, \\ Bunkyo-ku, Tokyo 113, Japan
}

Received November 15, 1989

\begin{abstract}
The effective thermal conductivity of air-impregnated gels of various air and water contents was measured at $20^{\circ} \mathrm{C}$ by the steady heat flow method, and the applicability of the sphere dispersion heat conduction model (the Maxwell-Eucken model) was studied. The Maxwell-Eucken model gave a fairly good approximation for the effective thermal conductivity of air-impregnated gels of the lowest water content, but that of air-impregnated gels of higher water content was underestimated by the Maxwell-Eucken model. In addition to that, the effective thermal conductivity of the air-impregnated gels of the highest water content at $40^{\circ} \mathrm{C}$ exceeded the maximum limit expected by the parallel layers heat conduction model. These facts suggested the existence of some heat transfer mechanism other than heat conduction and the significant role of water in it. To explain the discrepancy in applicability of known heat conduction models, a model of heat transfer in air-impregnated gels, which includes heat transfer by water vapor, is presented and discussed.
\end{abstract}

Knowledge of the effective thermal conductivity of food materials is important not only for the optimum design of processing equipment, but also for the prediction and the control of various changes within thermally processed food materials. For baked products, however, information regarding the effective thermal conductivity is scarce and the systematic understanding of the data is not enough as shown in a recent review. ${ }^{1)}$

Baked products are a kind of porous food materials, which macroscopically consist of a wet solid phase as a continuous phase and a gas phase as a dispersed phase. ${ }^{2)}$ For such heterogeneous two-phase mixtures, many structural models of heat conduction through them have been proposed as reviewed by Murakami and Okos. ${ }^{3)}$ To select the most appropriate heat conduction model for porous food materials, the thermal conductivity of each phase should be known. However, it is difficult to evaluate the thermal conductivity of the solid phase of a real porous food, dough without containing air for example, though some effort in the estimation has been made. ${ }^{4)}$
This is one of the reasons why the systematic understanding of the effective thermal conductivity data of such porous food materials as baked products is not good enough. Another reason is the complexity of heat transfer mechanisms. Miles et $a l^{5)}$ pointed out that heat transfer in porous foods is a complicated process involving not only heat conduction but radiation, convection, and evaporation and condensation of vapor. Though the effective thermal conductivity compensated for the contributions of heat transfer mechanisms other than conduction is useful in heat transfer calculations, the contributions of such different mechanisms to the effective thermal conductivity should be distinguished and evaluated for the systematic understanding.

A possible approach to the systematic understanding of effective thermal conductivity data is to select the applicable heat conduction model in the first place using a model porous system, in which a solid phase of known thermal conductivity is contained and heat is transferred by conduction alone. For this purpose, air-impregnated gels of extremely 
low water content will do because the thermal conductivity of the gel phase can be directly measured and the low water content prevents evaporation of water. Convective and radiative heat transfer can be suppressed by choosing the conditions of measurement.

The objective of this study is to analyze the effects of air and water contents on the effective thermal conductivity of air-impregnated gels, and to find an appropriate heat conduction model for air-impregnated gels in which heat is transferred only by conduction. Since an air-impregnated gel can be considered as a two-phase dispersion system consisting of a gel as a continuous phase and air bubbles as dispersed spheres, the following sphere dispersion heat conduction model, the MaxwellEucken model, ${ }^{6}$ may be applicable to their effective thermal conductivity as in the case of paraffin- or oil-impregnated gels shown in our previous paper. ${ }^{7)}$

$$
\frac{\lambda_{\mathrm{e}}}{\lambda_{\mathrm{c}}}=\frac{\lambda_{\mathrm{d}}+2 \lambda_{\mathrm{c}}-2 \phi\left(\lambda_{\mathrm{c}}-\lambda_{\mathrm{d}}\right)}{\lambda_{\mathrm{d}}+2 \lambda_{\mathrm{c}}+\phi\left(\lambda_{\mathrm{c}}-\lambda_{\mathrm{d}}\right)}
$$

where $\lambda_{\mathrm{c}}$ is the thermal conductivity of the gel, $\lambda_{\mathrm{d}}$ that of air, $\lambda_{\mathrm{e}}$ the effective thermal conductivity, and $\phi$ the volume fraction of air. Therefore, the experimental data for the gels of various air and water contents are compared with the Maxwell-Eucken model, and heat transfer mechanisms in air-impregnated gels are also discussed.

\section{Materials and Methods}

Preparation of air-impregnated gels. Gelatin solution was prepared by dissolving gelatin powder (Nacalai Tesque, Inc.) in distilled water at $75^{\circ} \mathrm{C}$. Into this solution, fine air bubbles were dispersed with a mixer (Mixmaster, Sunbeam). As the temperature of the air-impregnated sol was reduced, bubble migration was retarded. Then the sol was packed in a container and stored overnight at $5^{\circ} \mathrm{C}$.

To make gels of lesser water contents, $70.6 \mathrm{~g}$ of gelatin powder was dissolved in $400 \mathrm{ml}$ of an aqueous glycerol solution of 30 to $85 \%$ (by volume) at $80^{\circ} \mathrm{C}$. Fine air bubbles were dispersed into this solution, and the airimpregnated sol was packed and stored as described above.

Agar-gelatin mixed gels were also used to make airimpregnated gels of the highest water content as follows.
Table I. Compositions and Thermal Conductivity Values (at $20^{\circ} \mathrm{C}$ ) OF Gels USED IN THIS STUDY

\begin{tabular}{|c|c|c|c|c|}
\hline \multicolumn{4}{|c|}{ Composition (wt \%) } & \multirow{2}{*}{$\begin{array}{c}i_{c} \\
(\mathrm{~W} / \mathrm{mK})\end{array}$} \\
\hline Water & Glycerol & Gelatin & Agar & \\
\hline 96.6 & - & 1.7 & 1.7 & 0.587 \\
\hline 82.7 & - & 17.3 & - & 0.548 \\
\hline 74.0 & - & 26.0 & - & 0.529 \\
\hline 65.3 & - & 34.7 & - & 0.501 \\
\hline 57.7 & 30.1 & 12.2 & - & 0.476 \\
\hline 40.0 & 48.3 & 11.7 & - & 0.413 \\
\hline 23.8 & 64.9 & 11.3 & - & 0.352 \\
\hline 12.4 & 76.7 & 10.9 & - & 0.323 \\
\hline
\end{tabular}

Agar powder (Nacalai Tesque, Inc.) was dissolved in distilled water by heating up to boiling with condensed water refluxing, followed by addition of gelatin powder at about $50^{\circ} \mathrm{C}$. Fine air bubbles were dispersed into this mixed solution, and the air-impregnated sol was packed and stored.

The gravimetric compositions of the gels used in this study were listed in Table I. They were compensated for the water contents of the original gelatin and agar powders measured by heating at $105^{\circ} \mathrm{C}$ for $3 \mathrm{hr}$.

Volume fraction of air. The volume fraction of air was calculated from the density of each gel.

Measurement of effective thermal conductivity. The effective thermal conductivity of each gel was measured by the steady heat flow method with a Shotherm RTM-G5 (Showa Denko Co., Ltd.). The details of the measurement were described elsewhere. ") The temperature gradient applied to the sample was between 250 and $500 \mathrm{~K} / \mathrm{m}$ in all measurements.

\section{Results}

Thermal conductivity values of gelatin gels and gelatin-glycerol gels without air bubbles were measured at $20^{\circ} \mathrm{C}$ and shown in Table I. The values in Table I were used as $\lambda_{\mathrm{c}}$ for the following analysis on effective thermal conductivity data of air-impregnated gels.

Figure 1 shows the experimental values of $\lambda_{\mathrm{e}} / \lambda_{\mathrm{c}}$ for air-impregnated gelatin gels at $20^{\circ} \mathrm{C}$. In this case of high water content, all of the experimental values were greater than the values expected by the Maxwell-Eucken model, and rather close to the values expected by the parallel layers heat conduction model, which is 


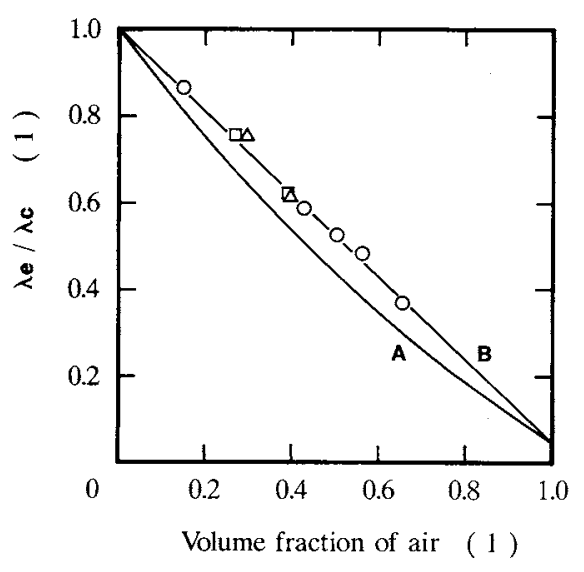

Fig. 1. Effective Thermal Conductivity of Air-impregnated Gelatin Gels (Gels of High Water Content) at $20^{\circ} \mathrm{C}$.

Water content of the gels: $\bigcirc, 82.7 \% ; \triangle, 74.0 \% ; \square, 65.3 \%$. Solid curves represent the Maxwell-Eucken model (A) and the parallel layers heat conduction model (B).

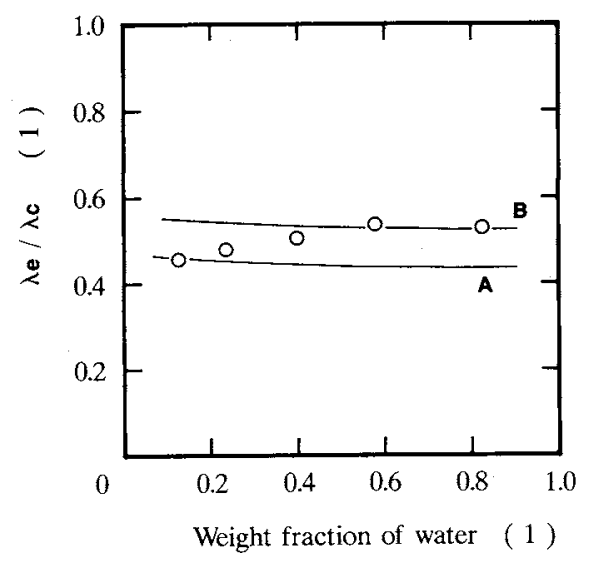

Fig. 2. Effects of Water Content on the Value of $\lambda_{\mathrm{c}} / \lambda_{\mathrm{c}}$ for Air-impregnated Gelatin and Gelatin-Glycerol Gels $(\phi=0.48-0.51)$ at $20^{\circ} \mathrm{C}$.

Solid curves represent the Maxwell-Eucken model (A) and the parallel layers heat conduction model (B).

represented by the following equation.

$$
\lambda_{\mathrm{e}}=\phi \lambda_{\mathrm{d}}+(1-\phi) \lambda_{\mathrm{c}}
$$

Figure 2 shows the effect of the water content of the gel phase on the value of $\lambda_{\mathrm{e}} / \lambda_{\mathrm{c}}$ for air-impregnated gels of $\phi \simeq 0.5$ at $20^{\circ} \mathrm{C}$. When the weight fraction of water was greater than 0.5 , the experimental value of $\lambda_{\mathrm{e}} / \lambda_{\mathrm{c}}$ was almost constant and approximated by the parallel

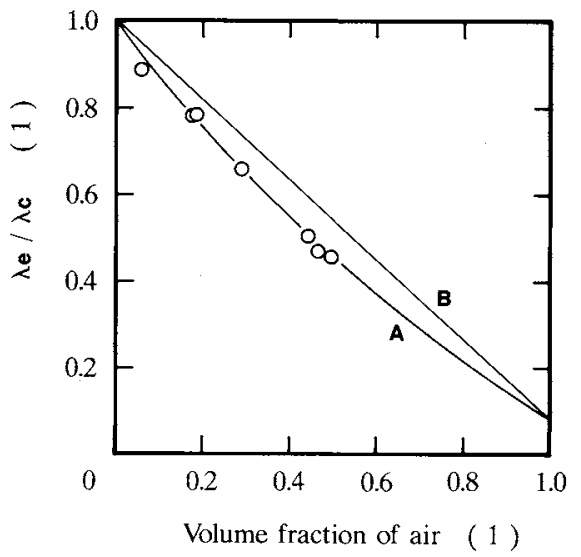

Fig. 3. Effective Thermal Conductivity of Air-impregnated Gelatin-Glycerol Gels of the Lowest Water Content $(12.4 \%)$ at $20^{\circ} \mathrm{C}$.

Solid curves represent the Maxwell-Eucken model (A) and the parallel layers heat conduction model (B).

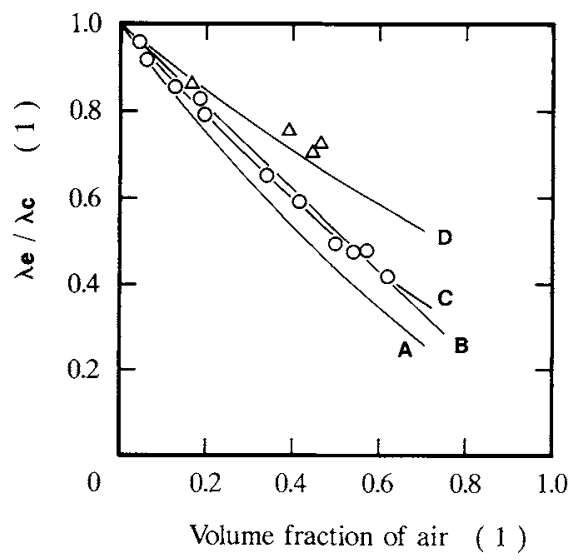

Fig. 4. Effective Thermal Conductivity of Air-impregnated Agar-Gelatin Mixed Gels Containing 96.6\% Water at $20^{\circ} \mathrm{C}(\bigcirc)$ and $40^{\circ} \mathrm{C}(\triangle)$.

Solid curves represent the Maxwell-Eucken model (A), the parallel layers heat conduction model (B) and Eq. (4) at $20^{\circ} \mathrm{C}(\mathrm{C})$ and $40^{\circ} \mathrm{C}(\mathrm{D})$.

layers heat conduction model as shown in Fig. 1. But when the weight fraction of water was below 0.5 , the experimental value of $\lambda_{\mathrm{e}} / \lambda_{\mathrm{c}}$ was on the decrease and got closer to the value expected by the Maxwell-Eucken model as the water content decreased. Thus, the effective thermal conductivity of air-impregnated gels of intermediate water content was in between the values expected from Eqs. (1) and (2). 
Figure 3 shows the experimental values of $\lambda_{\mathrm{e}} / \lambda_{\mathrm{c}}$ for air-impregnated gelatin-glycerol gels of the lowest water content $(12.4 \%)$ at $20^{\circ} \mathrm{C}$. It decreased with increase of the volume fraction of air and could be predicted fairly well by the Maxwell-Eucken model.

The effective thermal conductivity of airimpregnated agar-gelatin mixed gels containing $96.6 \%$ water was measured at $20^{\circ} \mathrm{C}$ and $40^{\circ} \mathrm{C}$ to show the effects of temperature, and the results are shown in Fig. 4. As in the case of air-impregnated gelatin gels shown in Fig. 1, the effective thermal conductivity of airimpregnated agar-gelatin mixed gels at $20^{\circ} \mathrm{C}$ was approximated by the parallel layers heat conduction model. Though the parallel layers heat conduction model gives the maximum limit of the effective thermal conductivity, the effective thermal conductivity of the airimpregnated gels measured at $40^{\circ} \mathrm{C}$ was much greater than this maximum limit.

\section{Discussion}

The thermal conductivity values of gelatin gels listed in Table I are slightly higher than those reported by Kong et al. ${ }^{87}$ and Andrieu $e t$ $a l .{ }^{9)}$ Both of them calculated the thermal conductivity of gelatin gels from the thermal diffusivity data obtained by the transient method. Aside from the difference in the method of measurements, the reason for this difference is not clear, but the purity of the gelatin used or the method of gel preparation may cause such a slight difference.

The value of $\lambda_{\mathrm{c}} / \lambda_{\mathrm{c}}$ was decreased with reducing water content, and the effective thermal conductivity of air-impregnated gels of the lowest water content could be predicted by the Maxwell-Eucken model. Since convective and radiative heat transfer was negligible under the experimental conditions used in this study and the lowest water content probably suppressed water migration, it was expected that heat flowed through such gels of the lowest water content only by conduction as in the case of the paraffin- or oil-impregnated gels. ${ }^{7)}$ Therefore, the results for the air-impregnated gels of the lowest water content suggest the applicability of the Maxwell-Eucken model for the prediction of the effective thermal conductivity of other air-impregnated food materials in which heat is transferred only by conduction.

For air-impregnated gels of higher water content, however, the effective thermal conductivity was underestimated by the Maxwell-Eucken model and approximated by the parallel layers heat conduction model as shown in Fig. 1. Compared with the case of the lowest water content, this suggests the effective thermal conductivity of those air-impregnated gels may involve the contribution of heat transfer mechanisms other than conduction. If heat flows through a mixture only by conduction, the effective thermal conductivity of the mixture must not exceed the maximum limit expected by the parallel layers heat conduction model. However, the effective thermal conductivity of air-impregnated gels of the highest water content measured at $40^{\circ} \mathrm{C}$ was much greater than this limit. This clearly indicates the existence of some heat transfer mechanism other than conduction. But convective and radiative heat transfer were negligible in these measurements. Considering the influence of the water content on the applicability of the Maxwell-Eucken model shown in Fig. 2, water must be concerned somehow in the heat transfer.

The heat transfer mechanism, in which water is concerned, may be considered as follows. According to the gradient of water vapor pressure caused by the temperature gradient, water vapor diffuses in a pore space. At the same time, evaporation and condensation of water take places at the interface of the pore. Thus the latent heat of evaporation of water is conveyed in the pore. Such heat transfer mechanism was already considered in other kinds of porous materials, namely snow, ${ }^{10}$ ) soil, ${ }^{11)}$ and packed beds of wet granular materials. ${ }^{12)}$ Also, in the baking process, the existence of the heat transfer mechanism mentioned above was pointed out. ${ }^{13)}$

The contribution of the heat transfer mech- 
anism mentioned above may be estimated as follows. The latent heat transfer accompanied by the water vapor diffusion can be evaluated as the following equivalent thermal conductivity. ${ }^{14)}$

$$
\lambda_{\mathrm{v}}=\frac{D}{R T} \frac{P}{P-P_{\mathrm{v}}} L \frac{\mathrm{d} P_{\mathrm{v}}}{\mathrm{d} T}
$$

where $\lambda_{\mathrm{v}}$ is the equivalent thermal conductivity due to the latent heat transfer, $D$ the diffusivity of water vapor in air, $L$ the latent heat of evaporation of water, $P$ total pressure, $P_{\mathrm{v}}$ partial pressure of water vapor, $R$ gas constant, and $T$ absolute temperature. Therefore, the overall thermal conductivity of the pore may be represented as the sum of $\lambda_{\mathrm{d}}$ and $\lambda_{\mathrm{v}}$. Then the effective thermal conductivity of airimpregnated gels of high water content may be evaluated using the Maxwell-Eucken model with this overall thermal conductivity of the pore. The replacement of $\hat{\lambda}_{\mathrm{d}}$ in Eq. (1) by $\lambda_{\mathrm{d}}+\lambda_{\mathrm{v}}$ results in the following equation.

$$
\frac{\lambda_{\mathrm{e}}}{\lambda_{\mathrm{c}}}=\frac{\left(\lambda_{\mathrm{d}}+\lambda_{\mathrm{v}}\right)+2 \lambda_{\mathrm{c}}-2 \phi\left\{\lambda_{\mathrm{c}}-\left(\lambda_{\mathrm{d}}+\lambda_{\mathrm{v}}\right)\right\}}{\left(\lambda_{\mathrm{d}}+\lambda_{\mathrm{v}}\right)+2 \lambda_{\mathrm{c}}+\phi\left\{\lambda_{\mathrm{c}}-\left(\lambda_{\mathrm{d}}+\lambda_{\mathrm{v}}\right)\right\}}
$$

Assuming $P_{\mathrm{v}}$ was equal to the saturated vapor pressure of water, Eq. (4) was illustrated by curves $C\left(20^{\circ} \mathrm{C}\right)$ and $D\left(40^{\circ} \mathrm{C}\right)$ in Fig. 4. As shown in Fig. 4, Eq. (4) gave a fairly good approximation for the effective thermal conductivity of air-impregnated gels of the highest water content, which suggests the adequacy of the heat transfer mechanism discussed above and the usefulness of Eq. (4).

On the basis of Eqs. (3) and (4), the influence of water content on the effective thermal conductivity shown in Fig. 2 can be explained as follows. With reducing water content, the value of $\lambda_{v}$ defined by Eq. (3) is decreased because of the decrease in vapor pressure of water. Therefore, according to Eq. (4), the value of $\lambda_{\mathrm{e}} / \lambda_{\mathrm{c}}$ is decreased and gets closer to that expected by the Maxwell-Eucken model as the water content decreased.

Equations (3) and (4) suggest that the effective thermal conductivity of air-impregnated gels of high water content depends greatly on temperature because of the great dependence of water vapor pressure on it. As concerns temperature, however, the conditions of thermal conductivity measurements in this study were rather limited because the melting temperature of the gels used in this study is low. For further discussion on the applicability of Eq. (4), measurements will be made over a wide range of temperature with other materials in a coming paper.

\section{Nomenclature}

$$
\begin{array}{rlr}
D & =\text { diffusivity of water vapor in air } & \left(\mathrm{m}^{2} / \mathrm{s}\right) \\
L= & \text { latent heat of evaporation of water } & (\mathrm{J} / \mathrm{mol}) \\
P= & (\mathrm{Pa}) \\
P_{\mathrm{v}}=\text { partial pressure of water vapor } & (\mathrm{Pa}) \\
R= & (\mathrm{J} / \mathrm{mol} \mathrm{K}) \\
T= & (\mathrm{K}) \\
\lambda= & (\mathrm{W} / \mathrm{mK}) \\
\lambda_{\mathrm{y}}= & \text { absormate temperature } & \\
& \text { latent heat transfer accompanied by water } \\
& \text { vapor diffusion } & (\mathrm{W} / \mathrm{mK}) \\
\phi= & \text { volume fraction of air }
\end{array}
$$

Subscripts

$\mathrm{c}=$ continuous phase material (gel)

$\mathrm{d}=$ dispersed phase material (air)

$\mathrm{e}=$ composite material (effective value)

\section{References}

1) C. Rask, J. Food Eng., 9, 167 (1989).

2) V. E. Sweat, in "Engineering Properties of Foods," ed. by M. A. Rao and S. S. H. Rizvi, Marcel Dekker, Inc., New York, 1986, pp. 49-87.

3) E. G. Murakami and M. R. Okos, in "Food Properties and Computer-Aided Engineering of Food Processing Systems," ed. R. P. Singh and A. G. Medina, Kluwer Academic Publishers, Dordrecht, 1989, pp. 3-48.

4) T. Tadano, Bull. Coll. Agric. Vet. Med., Nihon Univ., 44, 18 (1987).

5) C. A. Miles, G. van Beek and C. H. Veerkamp, in "Physical Properties of Foods," ed. by R. Jowitt, F. Escher, B. Hallström, H. F. Th. Meffert, W. E. L. Spiess and G. Vos, Applied Science Publishers, London, 1983, pp. 269-312.

6) A. Eucken, Forsch. Gebiete Ingenieur., 11, 6 (1940).

7) T. Sakiyama, Y. Matsushita, Y. Shiinoki and T. Yano, J. Food Eng., in press.

8) J.-Y. Kong, O. Miyawaki, K. Nakamura and T. Yano, Agric. Biol. Chem., 46, 783 (1982).

9) J. Andrieu, E. Gonnet and M. Laurent, in "Food Engineering and Process Applications," ed. by M. LeMaguer and P. Jelen, Elsevier Applied Science 
Publishers, London, 1986, pp. 103-122.

10) Y.-C. Yen, J, Geophys. Res,, 68, 1093 (1963).

11) M. Fukuda, B. D. Kay, H. Izuta and M. I. Sheppard, Dojo no Butsurisei, 49, 54 (1984).

12) M. Okazaki, I. Ito and R. Toei, AIChE Symp. Ser., 73, 164 (1977).
13) M. Nebelung, 1979, Ph. D. Thesis, TU Dresden, DDR. Cited in C. Rask, J. Food Eng., 9, 167 (1989).

14) A. W. Pratt, in "Thermal Conductivity," Vol. 1, ed. by R. P. Tye, Academic Press, London, 1969, pp. 301-405. 